\title{
Simultaneous Loss of Bilateral Voluntary Eyelid Opening and Sustained Winking Response Following Bilateral Posterior Cerebral Artery Infarction
}

\author{
Joon Yeop Kim, $\mathrm{MD}^{1,2}$, Yong Wook Kim, $\mathrm{MD}, \mathrm{PhD}^{1,2}$, Hyoung Seop Kim, $\mathrm{MD}^{3}$ \\ ${ }^{1}$ Department of Rehabilitation Medicine and Research Institute of Rehabilitation Medicine, \\ Yonsei University College of Medicine, Seoul; Department of Rehabilitation Medicine, Severance Hospital, Seoul; \\ ${ }^{2}$ Department of Physical Medicine and Rehabilitation, National Health Insurance Service Ilsan Hospital, Goyang, Korea
}

Spontaneous opening and closing of both eyes usually occurs in the normal awake state, unless a deliberate and voluntary attempt is made to open only one eye. We present a rare case of a male patient who was unable to open both eyes simultaneously after bilateral posterior cerebral artery infarction. He was able to close both eyes voluntarily. However, he was unable to keep both eyes open simultaneously and either the right or left eye remained closed. Upon a verbal command to open both eyes, the opened eye closed and the contralateral eye opened. When the closed eye was forced open, the opened eye closed. We thus presented a case of right-left dissociation of voluntary eyelid opening following bilateral posterior cerebral artery infarction, which was treated with botulinum toxin type A injection. Differential diagnosis to other movement disorders of the eyelids was discussed.

Keywords Eyelids, Botulinum toxins, Posterior cerebral artery, Blinking

\section{INTRODUCTION}

Blinking is a rapid eyelid movement that is essential to maintaining a healthy ocular surface and clarity of vision.

Received April 10, 2014; Accepted August 1, 2014

Corresponding author: Hyoung Seop Kim

Department of Physical Medicine and Rehabilitation, National Health Insurance Service Ilsan Hospital, 100 Ilsan-ro, Ilsan-donggu, Goyang 410-719, Korea

Tel: +82-31-900-0137, Fax: +82-31-900-0343, E-mail: rekhs@nhimc.or.kr

(c) This is an open-access article distributed under the terms of the Creative Commons Attribution Non-Commercial License (http://creativecommons. org/licenses/by-nc/3.0) which permits unrestricted noncommercial use, distribution, and reproduction in any medium, provided the original work is properly cited.

Copyright $\odot 2015$ by Korean Academy of Rehabilitation Medicine
Blinking can be spontaneous, reflex or voluntary [1].

The eyelid movements are mediated mainly by the orbicularis oculi (OrbOcu) and the levator palpebrae superioris (LPS) muscles. Eye closure and opening is achieved by the coordinated inhibition or activation of the LPS and OrbOcu muscles under the control of nuclei of the posterior commissure [2]. Both muscles have strictly balanced reciprocal innervation, whose derangement probably underlies many eyelid movement disorders [3]. Eyelid movement abnormalities may be related with viscosity of tears, or neuromuscular and peripheral nerve lesions. These conditions include myasthenia gravis, oculomotor nerve or facial nerve palsy and Graves' disease. Moreover, pathological eyelid movements can occur in central 
nervous system disorders, such as Parkinsonism, cerebrovascular disease, and multiple sclerosis.

Sole eyelid tonic closure associated with an inability to maintain both eyes opened voluntarily after bilateral posterior cerebral artery (PCA) infarction has not been previously reported. Symptoms were improved by botulinum toxin type A (BoNT-A) injection. We presented this interesting case with a review of the related literature to help discriminate the condition from other eyelid movement disorders.

\section{CASE REPORT}

An 83-year-old male patient visited our emergency clinic because of a mental change. The initial computed tomography perfusion showed markedly decreased perfusion in the left pons, midbrain, thalamus and PCA territory, and mildly decreased perfusion in the remaining area of the posterior circulation. These findings were suggestive of occlusion of the distal basilar artery. Therefore, he was treated with intra-arterial urokinase thrombolysis for the recanalization of the basilar artery and bilateral P1 segment. Brain diffusion-weighted magnetic resonance imaging showed PCA territory acute infarction (Fig. 1). A physical examination on transfer revealed good grades of motor strength in both the upper and lower extremities. However, he was unable to stand or sit alone for more than 2 minutes because of tremor, and could barely stand unassisted. Tremor from his trunk and both extremities was evident while moving or at rest. His cognitive status
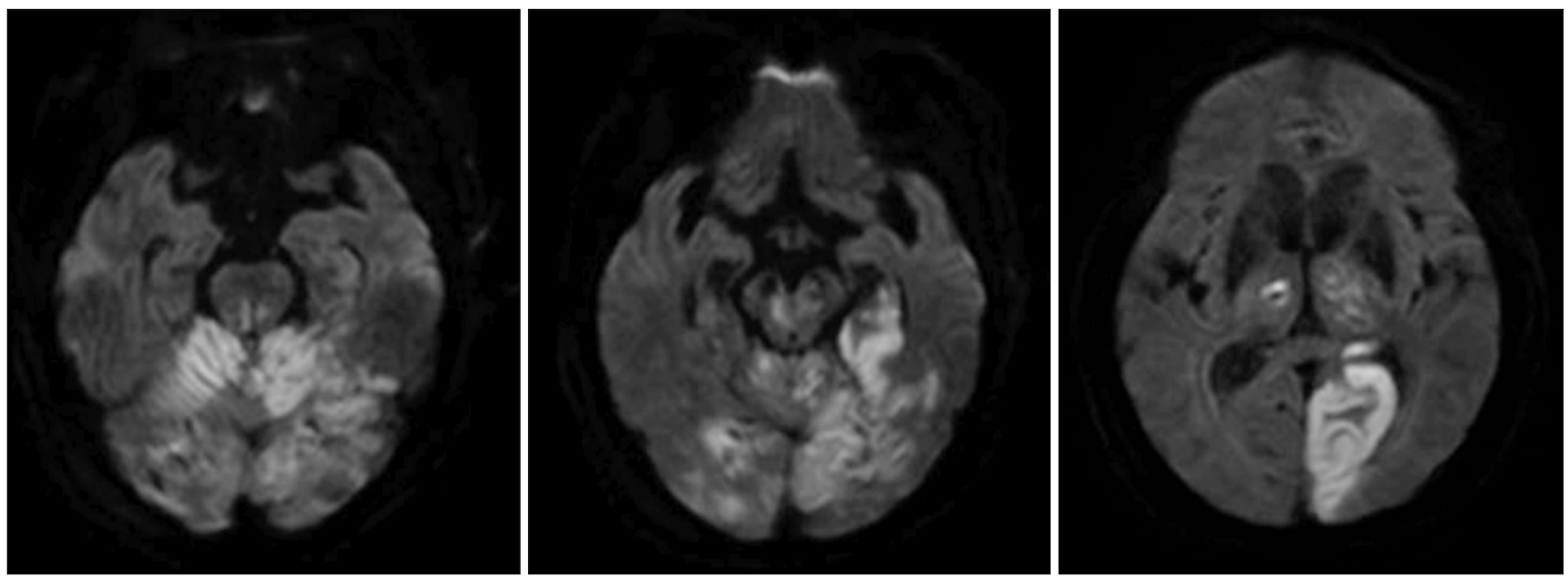

Fig. 1. Diffusion-weighted magnetic resonance image findings. Acute infarction at the level of pons, mid brain, bilateral cerebellum, bilateral thalamus and left visual cortex supplied by the basilar and posterior cerebellar arteries were evident.

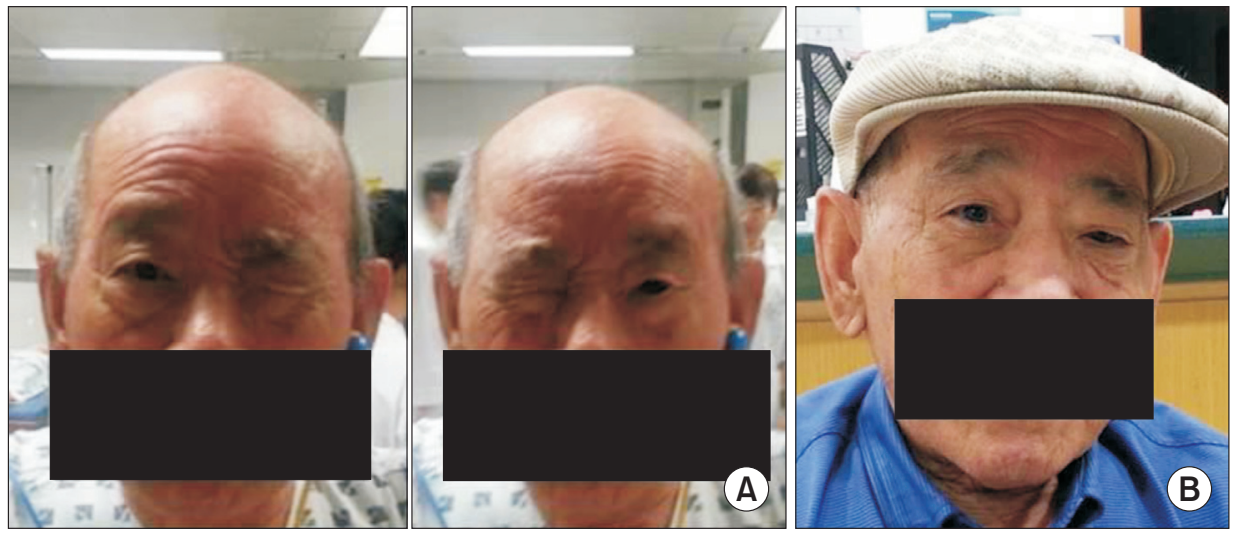

Fig. 2. (A) Before botulinum toxin type A injection, the patient showed a winking response, and was unable to open both eyes simultaneously. (B) After injection of $30 \mathrm{U}$ botulinum toxin type A into the right orbicularis oculi muscle, simultaneous opening and closing of both eyes was possible. 
was alert on the Korean-Mini Mental Status Examination that scored 14. He was able to obey 2-step commands. Speech evaluation showed a mild decay in vocal loudness, a harsh-strained voice quality and mild degree of dysarthria. Language evaluation using the Paradise Korean version of the Western Aphasia Battery (PK-WAB) showed an aphasia quotient of $72 \%$.

The patient was able to voluntarily close both eyes, but was unable to keep both eyes opened simultaneously; either the right or left eye was randomly kept closed (Fig. $2 \mathrm{~A})$. On verbal command to open an eye, the opened eye closed and the contralateral eye opened. Pursuant eye movements were unrestricted, the vestibulo-ocular reflex was preserved and both horizontal and vertical saccades were normal. There was no facial sensory change or weakness. When the closed eye was forced open by the examiner's finger, the examiner felt a forceful contraction of the OrbOcu muscle, and the opened eye closed automatically.

Visual evoked potential study stimulated both eyes individually and a scalp recording showed prolonged P100 latencies bilaterally (Fig. 3A). Blink reflex study showed
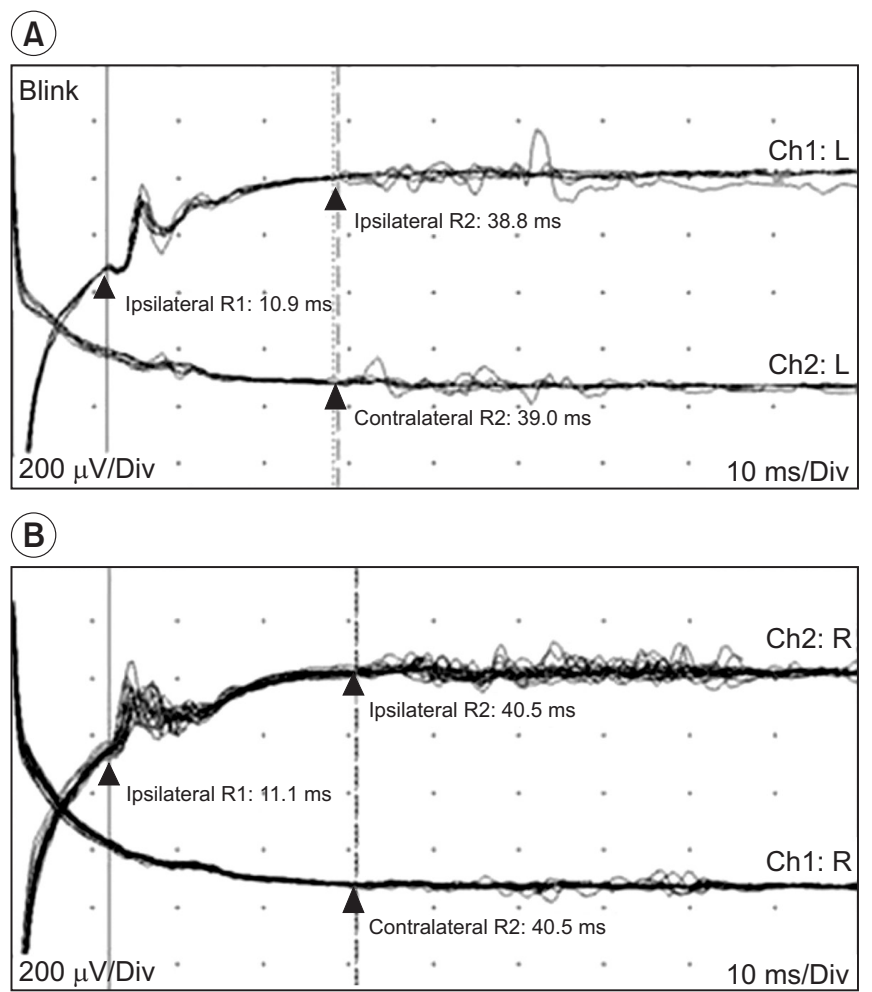

Fig. 3. (A, B) Blink reflex study showed normal findings at both sides. normal findings on both sides (Fig. 3B).

Clonazepam $2.0 \mathrm{mg}$ was administered prior to sleep to control tremors. Tremor of trunk and extremities almost disappeared post-medication, and the sitting and standing balance improved. Sitting and standing endurance also improved. However, abnormal eyelid movement persisted.

After discharge, the patient still complained about winking of his eyes. Bilateral eye tonic closure was presumably caused by focal dystonia of the OrbOcu muscles, and not by LPS weakness. Many reported cases of dystonia induced by cerebrovascular accident have been successful treated with botulinum toxin [4]. We therefore tried injection of BoNT-A 30U Botox (Allergan, Irvine, CA, USA) in the right OrbOcu muscle. The patient was capable of opening and closing both eyes simultaneously post-injection (Fig. 2B).

\section{DISCUSSION}

Blinking is categorized into three groups: spontaneous, reflex and voluntary blinking. Spontaneous blinking is part of an intrinsic system centrally regulated by different brainstem premotor structures via mainly dopaminergic activity. Reflex blinking is mainly via trigeminal, visual and acoustic afferent pathways, and the premotor areas at the pontine and medullary tegmental levels of the brainstem. Voluntary blinking is thought to be mediated by cortical areas close to the frontal eye fields [2]. Spontaneous and reflex blinking occurs through inhibition of the basal tonic LPS contraction by nuclei of the posterior commissure and concurrent activation of the OrbOcu muscles [2]. LPS inactivation precedes and outlasts OrbOcu activation. LPS tonic contraction with OrbOcu inactivation causes sustained eyelid opening, whereas LPS inactivation with OrbOcu contraction induces blinking and firm closure of the eyelids [3]. Disturbance of the intimate LPS-OrbOcu relationship can be categorized as three types: eyelid retraction-lagging, disturbance of blinking with eyelid postural maintenance, and the alteration of voluntary eyelid movements [3].

Eyelid retraction and lagging is associated with the periaqueductal grey matter close to the superior colliculus and the posterior commissure, which is important in eyelid closure. The lesions of these areas may cause LPS muscle overactivity and consequently eyelid retraction 
by the LPS muscle (Collier sign or Parinaud syndrome). It occurs with rostral dorsal midbrain lesion involving the pretectal region.

Disturbance of blinking and eyelid postural maintenance is thought to be induced by abnormalities in the LPS-OrbOcu relationship [3]. Generally, in spontaneous blinking and voluntary or reflex blinking, the total duration of OrbOcu activation is shorter than the LPS inhibition period. LPS inhibition precedes OrbOcu activation and persists longer. However, in pathologic conditions, such as Parkinson disease, focal dystonia, Balint syndrome and blepharospasm (BSP), this relationship is disturbed. For example, in focal dystonia, OrbOcu activity lasts longer than the LPS inhibitory period, which results in a partial co-contraction of antagonistic muscles. Also, for BSP, the mean span of OrbOcu activation persists when compared to patients without central motor eyelids disturbances, however, the span of LPS inhibition does not differ in both groups. This discrepancy is termed dystonic blinks.

Finally, the alteration of voluntary eyelid movements leads to failure in keeping the eyelids open and the inability to initiate eyelid elevation $[5,6]$. BSP is an example of excessive involuntary closure of the eyelids characterized by spasms of OrbOcu muscle contraction. Blepharocolysis (BCO) is an excessive involuntary closure of the eyelids characterized by prolonged inhibition of the LPS muscles in the absence of demonstrated OrbOcu contraction. Parkinson disease, cranial dystonia, and progressive supranuclear palsy exhibit combined BSP and BCO features that are associated with involuntary eyelid closure. Impairment of some pons and rostral midbrain area lesions is thought to be relevant to the BCO-BSP disorder. Furthermore, bilateral subthalamotomy reportedly provokes involuntary eyelid movement [7]. Hyperinhibition of LPS activation is associated with the complete loss of ongoing OrbOcu contraction in these BSP-BCO disorders [3]. Motor persistence of the OrbOcu muscle is another alteration of voluntary eyelid movements. It differs from BSP or BCO, because it is not related to involuntary drooping of the eyelids and the difficulty in opening the eyes only occurs after voluntary closure of the eyelids [8].

However, our case was different from former reported cases of eyelid movement disorder involving both eyes simultaneously. Our patient showed paradoxical eyelid movement involving winking, i.e., capable of closing both eyes simultaneously, but unable to open both eyes simultaneously. The patient showed an intact blink reflex indicative of an intact cranial nerve V-VII reflex, since the OrbOcu muscles are innervated by a facial nerve and the cranial nerve VII and LPS muscles are innervated by cranial nerve III. The results showed that the patient's peripheral nervous system that controls the OrbOcu and LPS muscles was intact and the pathomechanism was of central origin. Several previous studies were on patients with sustained eyelid closure with periaqueductal lesions [4]. The area ventral to the periaqueductal grey matter is related to eyelid closure, while the area dorsal to the periaqueductal grey matter is related to eye opening [3]. Therefore, we presumed that damage to the periaqueductal grey matter of the upper pons that is related to opening and closing both eyes led to impairment of simultaneous opening of both eyes [3].

Typically, simultaneous closing or opening of eyes bilaterally occurs without perception. After a cerebrovascular accident, many kinds of focal dystonia can occur in associated muscles. Tonic closure of eyelids developed bilaterally in the study subject. Thus, it represented a type of focal dystonia.

The ability of extremely low doses of BoNT-A to temporarily reduce muscle activity has found therapeutic application in the treatment of focal dystonia [9]. BoNTA is a well-known inhibitor of acetylcholine release from neuromuscular junctions. Abnormal tonicity of OrbOcu muscles induced by PCA territory injury might be resolved by BoNT-A injection.

The patient's symptom was bilateral but was resolved on unilateral BoNT-A injection in the right OrbOcu muscle. Our findings suggested that it was caused not only by forceful contraction of the OrbOcu muscle, but also by disconnection of some neural network within the PCA territory area. However, the exact pathophysiology was not apparent. This kind of symptom has seldom been reported. Correlation of symptoms with the neural network involved in eyelid movement requires further study.

\section{CONFLICT OF INTEREST}

No potential conflic of interest relevant to this article was reported. 


\section{REFERENCES}

1. Cruz AA, Garcia DM, Pinto CT, Cechetti SP. Spontaneous eyeblink activity. Ocul Surf 2011;9:29-41.

2. van Koningsbruggen MG, Peelen MV, Davies E, Rafal RD. Neural control of voluntary eye closure: a case study and an fMRI investigation of blinking and winking. Behav Neurol 2012;25:103-9.

3. Esteban A, Traba A, Prieto J. Eyelid movements in health and disease. The supranuclear impairment of the palpebral motility. Neurophysiol Clin 2004;34:315.

4. Schmidtke K, Büttner-Ennever JA. Nervous control of eyelid function: a review of clinical, experimental and pathological data. Brain 1992;115 Pt 1:227-47.

5. Esteban A, Gimenez-Roldan S. Involuntary closure of eyelids in parkinsonism: electrophysiological evi- dence for prolonged inhibition of the levator palpebrae muscles. J Neurol Sci 1988;85:333-45.

6. Defazio G, Livrea P, Lamberti P, De Salvia R, Laddomada G, Giorelli M, et al. Isolated so-called apraxia of eyelid opening: report of 10 cases and a review of the literature. Eur Neurol 1998;39:204-10.

7. Nashold BS Jr, Gills JP, Wilson WP. Ocular signs of brain stimulation in the human. Confin Neurol 1967;29:169-74.

8. Aramideh M, Ongerboer de Visser BW, Koelman $\mathrm{JH}$, Speelman JD. Motor persistence of orbicularis oculi muscle in eyelid-opening disorders. Neurology 1995;45:897-902.

9. Keam SJ, Muir VJ, Deeks ED. Botulinum toxin A (Dysport ${ }^{\circledR}$ ): in dystonias and focal spasticity. Drugs 2011;71:1043-58. 